\title{
Output Feedback Stabilization with Nonlinear Predictive Control: Asymptotic properties
}

\author{
Lars Imsland $^{\star}$, Rolf Findeisen ${ }^{\dagger}$, Frank Allgöwer ${ }^{\dagger}$, Bjarne A. Foss ${ }^{\star}$, \\ * Department of Engineering Cybernetics, Norwegian University of Science and Technology, \\ 7491 Trondheim, Norway \\ \{Lars.Imsland,Bjarne.Foss\}@itk.ntnu.no \\ $\dagger$ Institute for Systems Theory in Engineering, University of Stuttgart, 70550 Stuttgart, Germany \\ \{findeise, allgower\}@ist.uni-stuttgart.de
}

\begin{abstract}
State space based nonlinear model predictive control (NMPC) needs the state for the prediction of the system behavior. Unfortunately, for most applications, not all states are directly measurable. To recover the unmeasured states, typically a stable state observer is used. However, this implies that the stability of the closed-loop should be examined carefully, since no general nonlinear separation principle exists. Recently semi-global practical stability results for output feedback NMPC using a high-gain observer for state estimation have been established. One drawback of this result is that (in general) the observer gain must be increased, if the desired set the state should converge to is made smaller. We show that under slightly stronger assumptions, not only practical stability, but also convergence of the system states and observer error to the origin for a sufficiently large but bounded observer gain can be achieved.
\end{abstract}

Keywords: nonlinear model predictive control, output feedback, high-gain observers, asymptotic convergence

\section{Introduction}

Nonlinear model predictive control (NMPC) based on state space models is inherently a state feedback approach. Therefore, if not all states can be measured it is necessary to obtain state information from the output measurements using a state observer. While for linear systems, the separation principle implies that the stability of the closed-loop follows from the individual stability of the controller and the observer, a general separation principle for nonlinear systems does not exist. Thus, the stability of the closed-loop must be either enforced by design or checked afterwards. Checking the stability of the closed loop afterwards is often not possi- ble, since it does involve, for example, finding a suitable Lyapunov function for the closed loop system.

In the area of NMPC several researchers have addressed the output feedback problem. The approach in [3] derives local uniform asymptotic stability of contractive NMPC in combination with a "sampled" state estimator. In [10,11], see also [16], asymptotic stability results for observer based discrete-time NMPC for "weakly detectable" systems are given. The results allow, in principle, to estimate a (local) region of attraction of the output feedback controller from Lipschitz constants. However, it is in general not clear which parameters in the state feedback controller and observer should be changed to increase the region of attraction, or how to recover (in the limit) the region of attraction of the state feedback controller.

In contrast to these approaches, the control strategies derived in $[4-6,14]$ establish semi-global stability results, delivering direct tuning knobs to increase the resulting region of attraction of the closed-loop. The approach presented in [14] consist of an optimization based moving horizon observer combined with the so called dual-mode NMPC scheme proposed in [13]. Adding a contraction constraint to the moving horizon observer it is shown that the closed-loop is (semiglobally) asymptotically stable. However, for the results to hold, it is assumed that no model-plant mismatch and no disturbances are present, and a global optimization problem for the moving horizon observer must be solved.

In [6] asymptotic stability for instantaneous NMPC using high-gain observers for state recovery is obtained. Instantaneous NMPC means that the open-loop optimal control problem appearing in the NMPC controller must be solved at every time instant, leading to instantaneous feedback. Since in general no closed/analytic solution to an open-loop optimal control problem can be found, the approach is not applicable in practice. 
For this reason the results have been expanded in $[4,5]$ to the general sampled-data NMPC case. In sampleddata NMPC the open-loop optimal control problem is only solved at discrete sampling instants and the resulting optimal input signal is applied open-loop in between. The key result obtained in $[4,5]$ establishes that the closed-loop is semi-global practically stable. Semiglobal practical stability in this context means, that for state initial conditions in any compact set contained in the region of attraction of the NMPC state feedback controller and for any size of the target set (containing the origin), there exists a minimum observer gain and a maximum sampling time such that the system and observer states will enter the desired target set in finite time. However, only practical stability is achieved. If the desired target set is decreased, the result implies that it is necessary to increase the observer gain and thus the observer speed further.

In this paper we build on the practical stability results derived in [5]. We show that under strengthened assumptions on the system and the observer there exist a minimum observer gain and a maximum sampling time, such that the system and observer state actually asymptotically converges to the origin.

The paper is structured as follows: Section 2 introduces the system class and the observability assumption. In Section 3 we present the NMPC output feedback control strategy consisting of a high-gain state estimator and a NMPC state feedback controller. Section 3.3 reviews the semi-global practical stability results obtained in [5], while the main result, , conditions for asymptotic convergence of the system and observer state, is presented in Section 4.

\section{System class and observability assumption}

We consider nonlinear systems given by

$$
\dot{x}=f(x, u), \quad y=h(x)
$$

where $x \in \mathcal{X} \subset \mathbb{R}^{n}$ denotes the system state, $u \in \mathcal{U} \subset \mathbb{R}^{m}$ is the system input, $y \in \mathbb{R}^{p}$ is the measured output, and $\mathcal{X}, \mathcal{U}$ denote the constrained sets of allowed states and inputs. The sets $\mathcal{X}$ and $\mathcal{U}$ are such that $\mathcal{U} \subset \mathbb{R}^{p}$ is compact, $\mathcal{X} \subseteq \mathbb{R}^{n}$ is connected and $(0,0) \in \mathcal{X} \times \mathcal{U}$. With respect to the functions $f: \mathbb{R}^{n} \times \mathcal{U} \rightarrow \mathbb{R}^{n}$ and $h: \mathbb{R}^{n} \times \mathcal{U} \rightarrow \mathbb{R}^{p}$ we assume that they are sufficiently smooth. Furthermore, the origin is as stationary point, i.e. $f(0,0)=0$ and $h(0)=0$.

We will consider systems that are uniformly completely observable. Uniform complete observability is defined in terms of the observability map $\mathcal{H}$, which is given by successive differentiation of the output $y$ :

$Y^{\top}=\left[y_{1}, \dot{y}_{1}, \ldots, y_{1}^{\left(r_{1}\right)}, y_{2}, \ldots, y_{p}, \ldots, y_{p}^{\left(r_{p}\right)}\right]=\mathcal{H}(x, U)^{\top}$
Here $Y$ is the vector of output derivatives, and $U$ in general contains the input and in addition, a number of input derivatives. We will, however, restrict us to systems where $\mathcal{H}$ does not depend on the input, nor the derivatives.

Assumption 1 The system (1) is uniformly completely observable in the sense that there exists a set of indices $\left\{r_{1}, \ldots, r_{p}\right\}$ such that the mapping $Y=\mathcal{H}(x)$ depends only on $x$, is smooth with respect to $x$ and its inverse from $Y$ to $x$ is smooth and onto.

Note that the set of indices $\left\{r_{1}, \ldots, r_{p}\right\}$ is not necessarily unique, different mappings $\mathcal{H}$ might exist. The assumption that the observability map does not depend on the input derivatives is strong. The more general case is treated in [5], resorting to practical stability results instead of asymptotic stability.

\section{NMPC output feedback controller}

The NMPC output feedback controller consists of a high-gain observer for state estimation and an NMPC state feedback controller. No specific NMPC controller is specified, rather a set of assumptions is stated that the NMPC scheme must satisfy. In principle these assumptions can be satisfied by a series of NMPC schemes, such as quasi-infinite horizon NMPC [2], zero terminal constraint NMPC [12] and NMPC schemes utilizing control Lyapunov functions to obtain stability $[8,15]$.

\subsection{NMPC State Feedback}

In the framework of predictive control, the input is defined by the solution of an open-loop optimal control problem that is solved at sampling instants. Between the sampling instants the optimal input is applied open-loop. For simplicity we denote the sampling instants by $t_{i}$, with $t_{i}-t_{i-1}=\delta, \delta$ being the sampling time. For a given time $t, t_{i}$ should be taken as the nearest previous sampling instant $t_{i}<t$. The openloop optimal control problem solved at any $t_{i}$ is given by:

$$
\min _{\bar{u}(\cdot)} J\left(\bar{u}(\cdot) ; x\left(t_{i}\right)\right)
$$

subject to:

$$
\begin{aligned}
& \dot{\bar{x}}=f(\bar{x}, \bar{u}), \quad \bar{x}(\tau=0)=x\left(t_{i}\right) \\
& \bar{u}(\tau) \in \mathcal{U}, \quad \bar{x}(\tau) \in \mathcal{X} \quad \tau \in\left[0, T_{p}\right] \\
& \bar{x}\left(T_{p}\right) \in \mathcal{E} .
\end{aligned}
$$

The cost functional $J$ is defined over the control horizon $T_{p}$ by the stage cost $F$ and the terminal penalty $E$.

$$
J\left(\bar{u}(\cdot) ; x\left(t_{i}\right)\right):=\int_{0}^{T_{p}} F(\bar{x}(\tau), \bar{u}(\tau)) d \tau+E\left(\bar{x}\left(T_{p}\right)\right) .
$$


We assume that the NMPC scheme fits into the given frame and satisfies the following assumptions.

Assumption 2 There exists a simply connected region $\mathcal{R} \subseteq \mathcal{X} \subseteq \mathbb{R}^{n}$ ("region of attraction of the state feedback $N M P C ")$ with $0 \in \mathcal{R}$ such that:

1. The stage cost $F: \mathcal{X} \times \mathcal{U} \rightarrow \mathbb{R}$ is locally Lipschitz, satisfies $F(0,0)=0$, and is lower bounded by a class $\mathcal{K}$ function ${ }^{1} \alpha_{F}: \alpha_{F}(\|x\|+\|u\|) \leq$ $F(x, u) \quad \forall(x, u) \in \mathcal{X} \times \mathcal{U}$.

2. The optimal control $\bar{u}^{\star}(\tau ; x)$ is piecewise continuous and locally Lipschitz in $x$ in $\mathcal{R}$, uniformly in $\tau$. That is, for a given compact set $\Omega \subseteq \mathcal{R}\left\|\bar{u}^{\star}\left(\tau ; x_{1}\right)-\bar{u}^{\star}\left(\tau ; x_{2}\right)\right\| \leq L_{u}\left\|x_{1}-x_{2}\right\| \forall \tau \in$ $\left[0, T_{p}\right), x_{1}, x_{2} \in \Omega$, where $L_{u}$ denotes the Lipschitz constant of $\bar{u}^{\star}(\tau ; x)$ (as a function of $x$ ) in $\Omega$.

3. The value function, which is defined as the optimal value of the cost for every $x \in \mathcal{R} V(x):=$ $J\left(\bar{u}^{\star}(\cdot ; x) ; x\right)$ is Lipschitz for all compact subsets of $\mathcal{R}$ and $V(0)=0, V(x)>0$ for all $x \in \mathcal{R} /\{0\}$.

4. Along solution trajectories starting at a sampling instant $_{i}$ at $x\left(t_{i}\right) \in \mathcal{R}$, the value function satisfies

$$
\begin{aligned}
& V\left(\bar{x}\left(t_{i}+\tau\right)\right)-V\left(x\left(t_{i}\right)\right) \leq \\
& \quad-\int_{t_{i}}^{t_{i}+\tau} F\left(\bar{x}(s), \bar{u}^{\star}\left(s ; x\left(t_{i}\right)\right)\right) d s, 0 \leq \tau \leq T_{p} .
\end{aligned}
$$

To establish the stability result it is furthermore necessary that for any compact subset $\mathcal{S} \subset \mathcal{R}$ we can find a compact outer approximation $\Omega_{c}(\mathcal{S})$ that contains $\mathcal{S}$ and is invariant under the NMPC state feedback.

Assumption 3 For all compact sets $\mathcal{S} \subset \mathcal{R}$ there is at least one compact set $\Omega_{c}(\mathcal{S})=\{x \in \mathcal{R} \mid V(x) \leq c\}$ such that $\mathcal{S} \subset \Omega_{c}(\mathcal{S})$.

In the following we will denote level sets of $V$ by $\Omega_{c}$, where the index $c$ defines the level. Assumptions 2.1 and 2.4 are satisfied by many stabilizing NMPC schemes. In principle Assumptions 2.2, 2.3 and 3 can also be satisfied. However, checking them is in general difficult, see [5].

\subsection{High Gain State Estimation}

The system state is recovered by an high-gain observer. Application of the coordinate transformation $\zeta=\mathcal{H}(x)$, where $\mathcal{H}$ is the observability mapping, to the system (1) leads to the system in observability normal form in $\zeta$ coordinates

$$
\begin{aligned}
& \dot{\zeta}=A \zeta+B \phi(\zeta, u), \\
& y=C \zeta .
\end{aligned}
$$

\footnotetext{
${ }^{1} \mathrm{~A}$ continuous function $\alpha:[0, \infty) \rightarrow[0, \infty)$ is a class $\mathcal{K}$ function, if it is strictly increasing and $\alpha(0)=0$.
}

The matrices $A, B$ and $C$ have the following structure

$$
\begin{aligned}
& A=\operatorname{blockdiag}\left[A_{1}, \ldots A_{p}\right], A_{i}=\left[\begin{array}{ccccc}
0 & 1 & 0 & \cdots & 0 \\
0 & 0 & 1 & \cdots & 0 \\
\vdots & & & \vdots \\
0 & \cdots & \cdots & 0 & 1 \\
0 & \cdots & \cdots & \cdots & 0
\end{array}\right]_{r_{i} \times r_{i}} \\
& B=\operatorname{blockdiag}\left[B_{1}, \ldots, B_{p}\right], B_{i}=\left[\begin{array}{llll}
0 & \cdots & 0 & 1
\end{array}\right]_{r_{i} \times 1}^{\top} \\
& C=\operatorname{blockdiag}\left[C_{1}, \ldots, C_{p}\right], C_{i}=\left[\begin{array}{llll}
1 & 0 & \cdots & 0
\end{array}\right]_{1 \times r_{i}},
\end{aligned}
$$

and $\phi: \mathbb{R}^{n} \times \mathbb{R}^{m} \rightarrow \mathbb{R}^{p}$ is the "system nonlinearity" in observability normal form. The high-gain observer

$$
\dot{\hat{\zeta}}=A \hat{\zeta}+H_{\epsilon}(y-C \hat{\zeta})+B \hat{\phi}(\hat{\zeta}, u)
$$

allows recovery of the states $[1,17] \zeta$ from information of $y(t)$ assuming that

Assumption $4 \hat{\phi}$ in (4) is globally bounded.

The function $\hat{\phi}$ is the approximation of $\phi$ that is used in the observer. The observer gain matrix $H_{\epsilon}$ is given by $H_{\epsilon}=$ blockdiag $\left[H_{\epsilon, 1}, \ldots, H_{\epsilon, p}\right]$, with $H_{\epsilon, i}^{\top}=\left[\alpha_{1}^{(i)} / \epsilon, \alpha_{2}^{(i)} / \epsilon^{2}, \ldots, \alpha_{n}^{(i)} / \epsilon^{r_{i}}\right]$, where $\epsilon$ is the socalled high-gain parameter since $1 / \epsilon$ goes to infinity for $\epsilon \rightarrow 0$. The $\alpha_{j}^{(i)}$ s are design parameters and must be chosen such that the polynomials

$$
s^{n}+\alpha_{1}^{(i)} s^{n-1}+\cdots+\alpha_{n-1}^{(i)} s+\alpha_{n}^{(i)}=0, i=1, \ldots, p
$$

are Hurwitz.

Note that estimates obtained in $\zeta$ coordinates can be transformed back to the $x$ coordinates by $\hat{x}=\mathcal{H}^{-1}(\hat{\zeta})$.

\subsection{Semi-Global Practical Stability}

Before moving to the asymptotic convergence result, we briefly review the results given in $[4,5]$. Note that by Assumption 1 the observability map $\mathcal{H}$ does not depend on the input and its derivatives, thus we do not need the "observer resetting" as used in [5].

The overall output feedback control is given by the NMPC state feedback controller and a high-gain observer. The open-loop input is only calculated at the sampling instants using the state estimates of the observer. The observer itself operates continuously. Assuming that $\hat{x}\left(t_{i}\right) \in \mathcal{R}$, the input applied to the system is given by:

$$
u(t):=\bar{u}^{\star}\left(t-t_{i} ; \hat{x}\left(t_{i}\right)\right)
$$

where $\bar{u}^{\star}\left(\cdot ; \hat{x}\left(t_{i}\right)\right)$ is the optimal open-loop input signal of the NMPC optimal control problem (2) obtained at time $t_{i}$ using the state estimate $\hat{x}\left(t_{i}\right)$ for prediction. The estimated state $\hat{x}\left(t_{i}\right)$ is obtained by

$$
\hat{x}\left(t_{i}\right)=\mathcal{H}^{-1}\left(\hat{\zeta}\left(t_{i}\right)\right),
$$


where $\hat{\zeta}\left(t_{i}\right)$ is the high-gain observer state in observability normal form. Thus, in between sampling instants $t_{i}$ to $t_{i+1}$ an open-loop input is applied to the system.

Since the observer estimate is not bounded to the feasibility region $\mathcal{R}$ of the NMPC controller, and since the open-loop optimal control problem does not have a solution outside $\mathcal{R}$, we also have to define a valid input for $\hat{x}\left(t_{i}\right) \notin \mathcal{R}$. For simplicity, we do this by assigning $\bar{u}^{\star}(\tau ; x)=u_{f} \in \mathcal{U}$ for $x \in \mathcal{R}$. Thus $\bar{u}^{\star}(\cdot ; x)$ is defined and bounded for all $x \in \mathbb{R}^{n}$.

It is convenient to consider in the following the scaled observer error $\eta$,

$$
\eta=\left[\eta_{11}, \ldots, \eta_{1 r_{1}}, \ldots, \eta_{p 1}, \ldots, \eta_{p r_{p}}\right], \text { with } \quad \eta_{i j}:=\frac{\zeta_{i j}-\hat{\zeta}_{i j}}{\epsilon^{r_{i}-j}} .
$$

Hence $\hat{\zeta}=\zeta-D_{\epsilon} \eta$ with $D_{\epsilon}=\operatorname{blockdiag}\left[D_{\epsilon, 1}\right.$, $\left.D_{\epsilon, 2}, \ldots, D_{\epsilon, p}\right], D_{\epsilon, i}=\operatorname{diag}\left[\epsilon^{r_{i}-1}, \ldots, 1\right]$. The closedloop system in between sampling instants is then given by

$$
\begin{aligned}
\dot{x}(t) & =f\left(x(t), u\left(t-t_{i} ; \hat{x}\left(t_{i}\right)\right)\right) \\
\epsilon \dot{\eta}(t) & =A_{0} \eta(t)+\epsilon B g\left(t, x(t), x\left(t_{i}\right), \eta(t), \eta\left(t_{i}\right)\right)
\end{aligned}
$$

where the matrix $A_{0}=\epsilon D_{\epsilon}^{-1}(A-H C) D_{\epsilon}$ is independent of $\epsilon$ and where the function $g$ is defined as the difference between $\hat{\phi}$ and $\phi$,

$$
\begin{aligned}
& g\left(t, x(t), x\left(t_{i}\right), \eta(t), \eta\left(t_{i}\right)\right)= \\
& \quad \phi\left(\zeta(t), \bar{u}^{\star}\left(t-t_{i} ; \hat{x}\left(t_{i}\right)\right)\right)-\hat{\phi}\left(\hat{\zeta}(t), \bar{u}^{\star}\left(t-t_{i} ; \hat{x}\left(t_{i}\right)\right)\right) .
\end{aligned}
$$

Note that due to Assumption 1, $\eta(t)$ will be continuous also at sampling instants.

In the following the set $\mathcal{Q} \subset \mathbb{R}^{n}$ is a fixed compact set for the observer initial state $\hat{x}_{0}$, whereas $\Gamma_{\epsilon}:=\{\eta \in$ $\left.\mathbb{R}^{n} \mid W(\eta) \leq \rho \epsilon^{2}\right\}$ defines a set for the scaled observer error $\eta$ that directly depends on $\epsilon$. The quadratic form $W(\eta)$ is defined by $W(\eta):=\eta^{\top} P_{0} \eta$, where $P_{0}$ is the solution of the Lyapunov equation $P_{0} A_{0}+A_{0}^{\top} P_{0}=-I$. The constant $\rho$ is specified such that $\Gamma_{\epsilon}$ is reached and is invariant after a time $T_{\mathcal{Q}}(\epsilon)$, where $T_{\mathcal{Q}}(\epsilon) \rightarrow 0$ as $\epsilon \rightarrow 0$ (see [5] for details).

With this setup, it is shown in $[4,5]$ that the output feedback scheme can achieve practical stability: For any small set containing the origin, there exists an observer gain and a sampling time such that the trajectories converge to the set in finite time and stay inside the set.

\section{Theorem 1 (Semi-global practical stability)} Given arbitrary compact sets $\mathcal{Q} \subset \mathbb{R}^{n}$ and $\mathcal{S} \subset \mathcal{R}$. Then, for any $\sigma>0$, there exists an $\epsilon_{1}^{\star}>0$ and $a \delta_{1}^{\star}>0$ such that for all $0<\epsilon<\epsilon_{1}^{\star}, 0<\delta<\delta_{1}^{\star}$, and all $\left(x_{0}, \eta_{0}\right) \in \mathcal{S} \times \mathcal{Q}$, the trajectories $(x(t), \eta(t))$ stay bounded, converge in finite time to the set $\|(x, \eta)\| \leq \sigma$, and $x(t) \in \mathcal{R} \forall t \geq 0$.
Note that neither convergence of the states nor the observer error to the origin is implied. Convergence to the set $\{(x, \eta) \mid\|(x, \eta)\| \leq \sigma\}$ is obtained by showing that the state and the observer reaches an invariant set $\Omega_{\alpha} \times \Gamma_{\epsilon} \subset\{(x, \eta) \mid\|(x, \eta)\| \leq \sigma\}$. For proving the main result, we will use Theorem 1 to place ourselves in such a set.

\section{Asymptotic Convergence}

The main contribution of this paper is to show that under slightly strengthened assumptions we can assure asymptotic convergence. To obtain the result we have to strengthen the assumptions on the observer, to assure convergence of the observer error to the origin.

Assumption 5 For $(x(t), \eta(t)) \in \Omega_{\alpha} \times \Gamma_{\epsilon}$, there exists a $L_{g}>0$ such that

$$
\left\|g\left(t, x(t), x\left(t_{i}\right), \eta(t), \eta\left(t_{i}\right)\right)\right\| \leq L_{g}\|\eta(t)\| .
$$

The above is true if the observer nonlinearity is the same as in the real system ${ }^{2}$.

The following lemma says that if the observer error is nonzero at a sampling instant, it will not become zero in the following sample period. This is used to show that the Lipschitz continuity of the control in the observer error at sample instants (which follows from Assumption 2) can be replaced with Lipschitz continuity of the control in the "present" observer error.

Lemma 4.1 For $\left(x\left(t_{i}\right), \eta\left(t_{i}\right)\right) \in \Omega_{\alpha} \times \Gamma_{\epsilon}$, and for $\tau \in$ $\left[t_{i}, t_{i+1}\right]$,

$$
\|\eta(\tau)\| \geq L_{\eta}\left\|\eta\left(t_{i}\right)\right\|
$$

for some $L_{\eta}>0$.

Assumption 5 is a prerequisite for this result. The proof, omitted here for brevity, can be found in [7].

The following lemma holds since we know (due to Theorem 1) that the states and observer error stay in a bounded set:

Lemma 4.2 Consider the trajectories of system (1) driven by the NMPC open-loop control law based on the correct state $x_{0}$ (state feedback) and the state estimate $\hat{x}_{0}=\mathcal{H}^{-1}\left(\mathcal{H}\left(x_{0}\right)-D_{\epsilon} \eta_{0}\right)$ (output feedback)

$$
\dot{x}(\tau)=f\left(x(\tau), \bar{u}^{\star}\left(\tau ; \hat{x}_{0}\right)\right), \quad \dot{\bar{x}}(\tau)=f\left(\bar{x}(\tau), \bar{u}^{\star}\left(\tau ; x_{0}\right)\right),
$$

\footnotetext{
${ }^{2}$ By Assumption 4, this implies that $\phi$ must be globally bounded. However, this is not a real limitation. Since Assumption 5 merely has to hold on a compact set, the nonlinearities only have to be equal on this set, and therefore we can bound the observer nonlinearity outside a compact set of interest.
} 
starting at $x(t)=\bar{x}(t)=x_{0}$, with $(x(\tau), \eta(\tau)) \in \Omega_{\alpha} \times \Gamma_{\epsilon}$ and $\bar{x}(\tau) \in \Omega_{\alpha}$ for all $t \leq \tau \leq t+T$, for some $T<T_{p}$. Then, there exist constants $L_{1}$ and $L_{2}$ such that

$$
\begin{array}{r}
\left|\int_{t}^{t+T} F\left(x(\tau), \bar{u}^{\star}\left(\tau ; \hat{x}_{0}\right)\right) d \tau-\int_{t}^{t+T} F\left(\bar{x}(\tau), \bar{u}^{\star}\left(\tau ; x_{0}\right)\right) d \tau\right| \\
\leq L_{1} \int_{t}^{t+T}\|\eta(\tau)\| d \tau
\end{array}
$$

and

$$
|V(x(t+T))-V(\bar{x}(t+T))| \leq L_{2} \int_{t}^{t+T}\|\eta(\tau)\| d \tau .
$$

The proof can be found in [7]. Given Lemma 4.1 and the Lipschitz continuity of the value function (Assumption 2.3), it is very similar to [5, Lemma 2], and hence omitted here.

With these results, asymptotic convergence of the closed-loop can be established.

Theorem 2 (Asymptotic Convergence) Given arbitrary compact sets $\mathcal{Q} \subset \mathbb{R}^{n}$ and $\mathcal{S} \subset \mathcal{R}$. Then there exists an $\epsilon_{2}^{\star}>0$ and $a \delta_{2}^{\star}>0$ such that for all $0<\epsilon<\epsilon_{2}^{\star}$, $0<\delta<\delta_{2}^{\star}$, and all $\left(x_{0}, \eta_{0}\right) \in \mathcal{S} \times \mathcal{Q}$, the trajectories $(x(t), \eta(t))$ stay bounded, $x(t) \in \mathcal{R} \forall t \geq 0$ and $(x(t), \eta(t)) \rightarrow(0,0)$ for $t \rightarrow \infty$.

Proof: $\quad$ Let $0<\tilde{\epsilon}_{2}$ be such that

$$
L_{1}+L_{2}+\frac{L_{g}\left\|P_{0}\right\|}{\sqrt{\lambda_{\min }\left(P_{0}\right)}} \leq \frac{1}{4 \tilde{\epsilon}_{2} \sqrt{\left\|P_{0}\right\|}} .
$$

Let $t^{\prime}, \tilde{\epsilon}_{1}$ and $\tilde{\delta}_{1}$ be given according to Theorem 1 such that for a $0<\delta \leq \tilde{\delta}_{1}$, there exist $0<\epsilon \leq \tilde{\epsilon}_{1}$ such that the trajectories are confined to a set $\Omega_{\alpha} \subset \mathcal{S}$ for $t>t^{\prime}$. Set $\epsilon_{2}^{\star}:=\min \left(\tilde{\epsilon}_{1}, \tilde{\epsilon}_{2}\right)$, and set $\delta_{2}^{\star}=\tilde{\delta}_{1}$. Below, we consider the sampling instant $t_{i}>t^{\prime}$ closest to $t^{\prime}$.

The proof consists of three parts. In the first part we establish a bound on the decrease of the observer error. In the second part we construct a Lyapunov-like function which is shown to decrease over one sampling interval. Repeatedly applying this result we obtain in the third part convergence to the origin.

First part (Bounding the observer error decrease): First we establish a bound for the decrease of the observer error in integral form. Inspired by [1] it is convenient to express this decrease by the square root of $W$. It follows that ${ }^{3}$

$$
\begin{gathered}
\frac{d}{d t} \sqrt{W(\eta(t))}=\frac{\partial W}{\partial \eta}\left[\frac{1}{\epsilon} A_{0} \eta(t)+B g(t, \cdot)\right] \frac{1}{2 \sqrt{W(\eta(t))}} \\
\leq-\frac{1}{2 \epsilon \sqrt{\left\|P_{0}\right\|}}\|\eta(t)\|+\frac{\left\|P_{0}\right\|\|\eta(t)\|\|g(t, \cdot)\|}{\sqrt{\lambda_{\min }\left(P_{0}\right)}\|\eta(t)\|} \\
\leq-\frac{1}{2 \epsilon \sqrt{\left\|P_{0}\right\|}}\|\eta(t)\|+\frac{L_{g}\left\|P_{0}\right\|}{\sqrt{\lambda_{\min }\left(P_{0}\right)}}\|\eta(t)\|,
\end{gathered}
$$

\footnotetext{
${ }^{3}$ For brievety, $g(t, \cdot)$ are used for $g\left(t, x(t), x\left(t_{i}\right), \eta(t), \eta\left(t_{i}\right)\right)$.
}

where both terms on the right hand side are linear in $\|\eta(t)\|$. Integrating we obtain for any finite $0<T \leq \delta$,

$$
\begin{aligned}
& \sqrt{W\left(\eta\left(t_{i}+T\right)\right)}-\sqrt{W\left(\eta\left(t_{i}\right)\right)} \\
\leq & \int_{t_{i}}^{t_{i}+T}\left(-\frac{1}{2 \epsilon \sqrt{\left\|P_{0}\right\|}}\|\eta(\tau)\|+\frac{L_{g}\left\|P_{0}\right\|}{\sqrt{\lambda_{\min }\left(P_{0}\right)}}\|\eta(\tau)\|\right) d \tau .
\end{aligned}
$$

Second part (Decrease over one sampling interval): We use a Lyapunov like argument considering as a combined "Lyapunov function" $\tilde{V}(x, \eta):=V(x)+\sqrt{W(\eta)}$ for the closed loop system. Note that since $V$ and $W$ are continuous, also $\tilde{V}$ is continuous. Let $\bar{x}(t)$ be the state feedback trajectory, as in Lemma 4.2. Then, for some $0<T \leq \delta$,

$$
\begin{aligned}
& \tilde{V}\left(x\left(t_{i}+T\right), \eta\left(t_{i}+T\right)\right)-\tilde{V}\left(x\left(t_{i}\right), \eta\left(t_{i}\right)\right) \\
= & V\left(x\left(t_{i}+T\right)\right)-V\left(x\left(t_{i}\right)\right)+\sqrt{W\left(\eta\left(t_{i}+T\right)\right)}-\sqrt{W\left(\eta\left(t_{i}\right)\right)} \\
\leq & V\left(\bar{x}\left(t_{i}+T\right)\right)-V\left(x\left(t_{i}\right)\right)+\left|V\left(x\left(t_{i}+T\right)\right)-V\left(\bar{x}\left(t_{i}+T\right)\right)\right| \\
& +\sqrt{W\left(\eta\left(t_{i}+T\right)\right)}-\sqrt{W\left(\eta\left(t_{i}\right)\right)} .
\end{aligned}
$$

From Assumption 2.4, it follows that

$$
\begin{gathered}
\tilde{V}\left(x\left(t_{i}+T\right), \eta\left(t_{i}+T\right)\right)-\tilde{V}\left(x\left(t_{i}\right), \eta\left(t_{i}\right)\right) \\
\leq-\int_{t_{i}}^{t_{i}+T} F\left(\bar{x}(\tau), u\left(\tau ; x_{0}\right)\right) d \tau \\
\quad+\left|V\left(x\left(t_{i}+T\right)\right)-V\left(\bar{x}\left(t_{i}+T\right)\right)\right| \\
\quad+\sqrt{W\left(\eta\left(t_{i}+T\right)\right)}-\sqrt{W\left(\eta\left(t_{i}\right)\right)} \\
\leq-\int_{t_{i}}^{t_{i}+T} F\left(x(\tau), u\left(\tau ; \hat{x}_{0}\right)\right) d \tau \\
\quad+L_{1} \int_{t}^{t_{i}+T}\|\eta(\tau)\| d \tau+L_{2} \int_{t_{i}}^{t_{i}+T}\|\eta(\tau)\| d \tau \\
+\int_{t_{i}}^{t_{i}+T}\left(-\frac{1}{2 \epsilon \sqrt{\left\|P_{0}\right\|}}\|\eta(\tau)\|+\frac{L_{g}\left\|P_{0}\right\|}{\sqrt{\lambda_{\min }\left(P_{0}\right)}}\|\eta(\tau)\|\right) d \tau
\end{gathered}
$$

where Lemma 4.2 is used in the last transition.

Hence, by the definition of $\epsilon_{2}^{\star}$, we have that for $0<\epsilon \leq$ $\epsilon_{2}^{\star}, 0<T \leq \delta_{2}$,

$$
\begin{aligned}
& \tilde{V}\left(x\left(t_{i}+T\right), \eta\left(t_{i}+T\right)\right)-\tilde{V}\left(x\left(t_{i}\right), \eta\left(t_{i}\right)\right) \\
& \leq-\int_{t_{i}}^{t_{i}+T} F\left(x(\tau), u\left(\tau ; \hat{x}_{0}\right)\right) d \tau-\int_{t_{i}}^{t_{i}+T} \frac{1}{4 \epsilon \sqrt{\left\|P_{0}\right\|}}\|\eta(\tau)\| d \tau .
\end{aligned}
$$

In particular this holds for $T=\delta$.

Third part (Convergence): Since, by Theorem 1, we will not leave the set $\Omega_{\alpha} \times \Gamma_{\epsilon}$, the above holds for all remaining sample intervals (using the same $\epsilon$ and $\delta$ ). This shows that $\tilde{V}$ is non-increasing. Together with continuity and the fact that $\tilde{V}$ is bounded from below, this implies that the limit $\tilde{V}(x(\infty), \eta(\infty))$ exists. By 
adding all sample intervals together, we obtain in the limit

$$
\begin{aligned}
& \tilde{V}(x(\infty), \eta(\infty))-\tilde{V}\left(x\left(t_{i}\right), \eta\left(t_{i}\right)\right) \\
\leq & -\int_{t_{i}}^{\infty} F\left(x(\tau), u\left(\tau ; \hat{x}_{0}\right)\right) d \tau-\int_{t_{i}}^{\infty} \frac{1}{4 \epsilon \sqrt{\left\|P_{0}\right\|}}\|\eta(\tau)\| d \tau \\
\leq & -\int_{t_{i}}^{\infty} \alpha_{F}(\|x(\tau)\|)+\frac{1}{4 \epsilon \sqrt{\left\|P_{0}\right\|}}\|\eta(\tau)\| d \tau .
\end{aligned}
$$

This implies that the infinite integral on the right hand side exists and is finite. Noting the continuity (in time) of the involved functions, convergence of $(x(t), \eta(t))$ to $(0,0)$ follows from Barbalat's lemma [9].

The result shows that there exists a finite observer gain such that the state and the observer asymptotically converge to the origin. Thus, under the assumptions made, it is not necessary to increase the observer gain to infinity to converge to a small desired region. Depending on the considered system, even relatively small observer gains might lead to asymptotic convergence.

\section{Conclusions}

The question of stability of output feedback NMPC is of practical as well as of theoretical relevance. In this paper we have extended the results derived in $[4,5]$. We showed that under certain conditions an output feedback NMPC controller based on the state estimate of a high-gain observer does lead to semi-global asymptotic convergence. For any desired compact set of initial conditions that is a subset of the region of attraction of the NMPC state feedback controller, there exists a minimum observer gain and a maximum sampling time, such that the states and the observer error asymptotically converge to the origin. Thus for reaching the origin, it is not necessary to increase the observer gain to infinity. Depending on the system considered, even small observer gains might lead to asymptotic convergence. This is of special interest, since a decrease of the error resulting from the state estimate does in general lead to an increases of robustness margins to other disturbances of the closed-loop.

\section{References}

[1] A.N. Atassi and H.K. Khalil. A separation principle for the stabilization of a class of nonlinear systems. IEEE Trans. Automatic Control, 44(9):16721687, 1999.

[2] H. Chen and F. Allgöwer. A quasi-infinite horizon nonlinear model predictive control scheme with guaranteed stability. Automatica, 34(10):1205-1218, 1998.

[3] S. de Oliveira Kothare and M. Morari. Contractive model predictive control for constrained nonlinear systems. IEEE Trans. Automat. Contr., 45(6):10531071, 2000.

[4] R. Findeisen, L. Imsland, F. Allgöwer, and B.A. Foss. Output feedback nonlinear predictive control - a separation principle approach. In Proceedings of 15 th IFAC World Congress, Barcelona, Spain, 2002.

[5] R. Findeisen, L. Imsland, F. Allgöwer, and B.A. Foss. Output feedback stabilization for constrained systems with nonlinear model predictive control. Int. J. of Robust and Nonlinear Control, 13(3-4):211-227, 2003.

[6] L. Imsland, R. Findeisen, E. Bullinger, F. Allgöwer, and B.A. Foss. A note on stability, robustness and performance of output feedback nonlinear model predictive control. To appear in J. Proc. Contr., 2002.

[7] L. S. Imsland. Topics in Nonlinear Control - Output Feedback Stabilization and Control of Positive Systems. PhD thesis, Norwegian University of Science and Technology, Department of Engineering Cybernetics, 2002.

[8] A. Jadbabaie, J. Yu, and J. Hauser. Unconstrained receding horizon control of nonlinear systems. IEEE Trans. Automat. Contr., 46(5):776 -783, 2001.

[9] H. K. Khalil. Nonlinear Systems. Prentice Hall, Upper Saddle River, NJ, second edition, 1996.

[10] L. Magni, D. De Nicolao, and R. Scattolini. Output feedback receding-horizon control of discrete-time nonlinear systems. In Preprints of the 4th Nonlinear Control Systems Design Symposium 1998 - NOLCOS'98, pages 422-427. IFAC, July 1998.

[11] L. Magni, G. De Nicolao, and R. Scattolini. Output feedback and tracking of nonlinear systems with model predictive control. Automatica, 37(10):1601$1607,2001$.

[12] D.Q. Mayne and H. Michalska. Receding horizon control of nonlinear systems. IEEE Trans. Automat. Contr., 35(7):814-824, 1990.

[13] H. Michalska and D.Q. Mayne. Robust receding horizon control of constrained nonlinear systems. IEEE Trans. Automat. Contr., AC-38(11):1623-1633, 1993.

[14] H. Michalska and D.Q. Mayne. Moving horizon observers and observer-based control. IEEE Trans. Automat. Contr., 40(6):995-1006, 1995.

[15] J. Primbs, V Nevistić, and J. Doyle. Nonlinear optimal control: A control Lyapunov function and receding horizon perspective. Asian Journal of Control, 1(1):14-24, 1999.

[16] P.O.M. Scokaert, J.B. Rawlings, and E.S. Meadows. Discrete-time stability with perturbations: Application to model predictive control. Automatica, 33(3):463-470, 1997.

[17] A. Tornambè. Output feedback stabilization of a class of non-miminum phase nonlinear sysems. Syst. Contr. Lett., 19(3):193-204, 1992. 